\title{
Avaliação de Treinamento em Entrevistas Investigativas com Crianças Vítimas de Violência Sexual
}

\author{
Carlos Aznar-Blefari ${ }^{1}$, Marcela Schimaneski Lunardon ${ }^{2}$, \\ Clarissa Pinto Pizarro de Freitas ${ }^{3}$, Luísa Fernanda Habigzang ${ }^{4}$
}

\begin{abstract}
http://orcid.org/0000-0001-8665-5304 / Pontifícia Universidade Católica do Rio Grande do Sul (PUCRS), Brasil
${ }^{2}$ http://orcid.org/0000-0001-8080-089X / Pontifícia Universidade Católica do Rio Grande do Sul (PUCRS), Brasil

${ }^{3}$ http://orcid.org/0000-0002-2274-8728 / Pontifícia Universidade Católica do Rio de Janeiro (PUC-Rio), Brasil

${ }^{4}$ http://orcid.org/0000-0002-0262-0356 / Pontifícia Universidade Católica do Rio Grande do Sul (PUCRS), Brasil
\end{abstract}

Resumo

Este estudo teve como objetivo avaliar o impacto de um programa de treinamento para o uso do protocolo do National Institute of Child Health and Human Development (NICHD). O treinamento foi composto por $20 \mathrm{~h}$ de atividades presenciais teórico-práticas. Participaram do estudo 55 profissionais com idade média de 38,4 anos. Inicialmente, foram mensurados os níveis de impacto do treinamento, engajamento no trabalho, autoeficácia ocupacional e características laborais. Em seguida, foram conduzidas entrevistas com seis participantes que, após o treinamento, adotaram a utilização do Protocolo NICHD ou optaram por não adotar a técnica. Os resultados apontam que os profissionais que participaram do treinamento o avaliaram de forma positiva e perceberam o impacto na sua prática profissional. Foi possível identificar que os níveis de engajamento no trabalho predizem a reação ao treinamento e antecedem a transferência de aprendizagem e o impacto do treinamento (i.e., transferência de treinamento).

Palavras-chave: avaliação de treinamentos, protocolo NICHD, impacto do treinamento.

\section{Evaluation of Training in Investigative Interviews with Child Victims of Sexual Violence}

Abstract

This study aimed to evaluate the impact of a training program for the use of the National Institute of Child Health and Human Development (NICHD) protocol. The training consisted of 20 hours of classroom activities. The study included 55 professionals with an average age of 38.4 years. Initially, the levels of training impact, work engagement, occupational self-efficacy and job characteristics were measured. Then, interviews were conducted with six participants who, after training, either adopted the use of the NICHD protocol, or did not adopt it. The results show that the levels of work engagement predict the satisfaction with the training. When analysed together, these two factors precede the transfer of learningand the impact of training.

Keywords: training evaluation, NICHD protocol, impact of training.

\section{Evaluación de Entrenamiento en Entrevistas de Investigación con Niños Víctimas de Violencia Sexual}

Resumen

Este estudio tuvo como objetivo evaluar el impacto de un programa de entrenamiento para el uso del protocolo del National Institute of Child Health and Human Development (NICHD).El entrenamiento consistió en 20 horas de actividades teórico-práctcas en el aula. Participaron del estudio 55 profesionales, con edad promedio de 38,4 años. Inicialmente, fueron medidos los niveles del impacto del entrenamiento, el compromiso laboral, la autoeficacia ocupacional y las características laborales. Luego, fueron realizadas entrevistas con seis participantes que, después del entrenamiento, adoptaron el uso del Protocolo NICHD o decidieron no adoptar la técnica. Los resultados muestran que los profesionales que participaron del entrenamiento lo evaluaron de forma positiva y percibieron el impacto em su practaica professional. Fue posible identificar que los niveles de compromisso laboral predicen la reacción al entrenamiento y anticipam la transferencia de aprendizaje y el impacto del entrenamiento (i.e, transferencia del entrenamiento).

Palabras clave: evaluación de entrenamiento, protocolo NICHD, impacto del entrenamiento. 
A complexidade na obtenção de provas relevantes à tomada de decisão judicial em casos de violência sexual (VS) contra crianças e adolescentes vem sendo discutida pela literatura há muitos anos. A VS, na maior parte dos casos, não deixa provas materiais do crime, não há testemunhas e o agressor dificilmente revela a situação temendo as consequências de seus atos. Desta forma, a principal fonte de informações sobre o evento em questão é o relato da vítima. Inúmeras pesquisas foram desenvolvidas sobre os aspectos necessários para a condução adequada de entrevistas investigativas (Lamb, Brown, Hershkowitz, Orbach, \& Esplin, 2018; Lamb, Hershkowitz, Orbach, \& Esplin, 2008; Williams, Padilha, Hackbarth, Blefari, \& Peixoto, 2014). Neste contexto, o desenvolvimento do protocolo do National Institute of Child Health and Human Develompent (NICHD) é considerado um marco na história das entrevistas investigativas no mundo (Faller, 2015). O protocolo NICHD foi desenvolvido em campo com base em aspectos desenvolvimentais de crianças e adolescentes (Lamb et al., 2008).

Profissionais treinados a utilizar o protocolo NICHD demonstram uma melhora na qualidade das entrevistas que conduzem (La Rooy et al., 2015). Este achado também é observado em contexto nacional (Aznar-Blefari \& Padilha, 2015; Hackbarth, Williams, \& Lopes, 2015). Com a adoção do protocolo NICHD são realizadas, em média, três vezes mais questões abertas (i.e., questões que não direcionam o relato da criança e que buscam informações baseadas em memória de evocação livre) em comparação a outras técnicas (Lamb et al., 2008; Lamb et al., 2018; La Rooy et al., 2015).

No Brasil, a Lei no 13.431 (Brasil, 2017) propõe mudanças importantes na legislação sobre a escuta de crianças e adolescentes com alegação de VS. A Lei cria o sistema de garantia de direitos e determina que os profissionais implicados na escuta dessas crianças e adolescentes sejam treinados, preferencialmente de maneira contínua e interdisciplinar. A Lei também descreve que o Depoimento Especial (i.e., escuta da criança por autoridade policial/ judicial) deve ser realizado por meio de protocolos de entrevista investigativa e sempre que possível será realizado apenas uma vez com a garantia da ampla defesa do investigado.

Há no Brasil importante carência de avaliações de treinamentos para profissionais que atendem vítimas de VS (Damásio, Habigznag, Freitas, \& Koller, 2014). Além disso, há poucos estudos empíricos nacionais demonstrando os efeitos de programas de treinamento na mudança de comportamento dos entrevistadores ao adotarem algum protocolo de entrevista investigativa (e.g., Aznar-Blefari \& Padilha, 2015; Hackbarth, Lopes, \& Williams, 2018). Internacionalmente, os estudos sobre o assunto, em geral, comparam a quantidade de questões adequadas (i.e., questões abertas) e inadequadas (i.e., questões de múltipla-escolha e sugestivas) antes e depois do treinamento (Lamb et al., 2008).

Em revisão de estudos sobre treinamentos de entrevista forense publicados entre 1999 e 2014, foram identificados 16 estudos com descrições sumárias dos conteúdos e planos de aula. A maioria destes estudos demonstrou que profissionais apresentam dificuldades em adotar técnicas recomendadas em sua prática profissional. Durante o período dos treinamentos, foi possível identificar um aumento no número de questões abertas utilizadas nas entrevistas dos participantes. Contudo, após a realização dos treinamentos, metade dos estudos avaliados demonstrou uma taxa igual ou inferior a 25\% de questões abertas (Benson \& Powell, 2015). A literatura aponta que o treinamento continuado por meio de revisões de pares ou supervisões contribuem para a manutenção dos níveis de questões abertas (Benson \& Powell, 2015; Lamb, 2016).

Há uma lacuna na literatura sobre o treinamento de profissio- nais que atuam realizando entrevistas investigativas, de pesquisas que avaliem outras variáveis que indicam o impacto do treinamento, tais como recursos pessoais (e.g., autoeficácia ocupacional, engajamento no trabalho) e recursos do trabalho (e.g., suporte à transferência de aprendizagem). Os mais tradicionais níveis de avaliação de treinamentos objetivam a mensuração da reação, da transferência de aprendizagem e do impacto (Hamblin, 1978; Kirkpatrick, 1976). Por reação compreende-se o nível de satisfação do participante em relação ao treinamento (Abbad, 1999; Abbad, Gama, \& Borges-Andrade, 2000). A transferência de aprendizagem é reconhecida como a aplicação do conhecimento aprendido de maneira formal (i.e., ações organizacionais de treinamento e desenvolvimento) ou informal no trabalho (Pilati \& Abbad, 2005; Sampaio, Borges-Andrade, \& Bonatti, 2018). Não se trata apenas de uma mensuração quantitativa de conteúdos aprendidos, mas sim a forma com que o participante assimila o conteúdo aprendido à sua prática profissional.

Por impacto (i.e., transferência de treinamento) compreende-se a generalização de habilidades desenvolvidas no treinamento após os profissionais retornarem ao trabalho (Schoeb, Lafrenière-Carrier, Lauzier, \& Courcy, 2019). Avalia-se neste ponto se o treinamento causou uma mudança no desempenho global do treinado, bem como em sua motivação e atitudes em relação ao trabalho (Pilati \& Abbad, 2005). Tal nível é descrito por Hamblin (1978) como impacto em amplitude. A crença do indivíduo sobre o quanto o treinamento pode ajudá-lo a melhorar sua atuação profissional pode atuar como fator motivacional para a realização da transferência do aprendizado (Curado, Henriques, \& Ribeiro, 2015).

Outra variável do contexto do trabalho que pode contribuir no impacto do treinamento (i.e., transferência de treinamento) é o suporte oferecido pela organização ao desenvolvimento das atividades de treinamento. Uma revisão narrativa de 100 anos de pesquisas publicadas no Journal of Applied Psychology (JAP) demonstrou uma relação direta entre o suporte organizacional (i.e., suporte dos pares e do supervisor) e a eficácia das atividades de treinamento. Os autores demonstram que o suporte oferecido pela organização e a cultura organizacional afetam a motivação dos profissionais para o aprendizado e consequentemente a transferência do treinamento (Bell, Tannenbaum, Ford, Noe, \& Kraiger, 2017).

Variáveis individuais podem influenciar a avaliação do treinamento e sua aplicabilidade pelos participantes. Dentre elas destacam-se o engajamento no trabalho e autoeficácia ocupacional. Ambas atuam como fatores protetivos à saúde do trabalhador e potencializam a transferência do aprendizado (Freitas, Silva, Damásio, Koller, \& Teixeira, 2014; Schaufeli, 2014). Por autoeficácia ocupacional entende-se as crenças dos profissionais relacionadas à percepção sobre a sua capacidade em realizar com sucesso, ou não, tarefas que auxiliem populações em situação de vulnerabilidade. É considerada um recurso pessoal no trabalho protetivo ao desenvolvimento de burnout e afetos negativos (Freitas, Damásio, \& Koller, 2017; Freitas et al., 2016)

O engajamento no trabalho é compreendido como um estado mental, disposicional, positivo e persistente de bem-estar e prazer intenso pela ação de seu trabalho. Trata-se de um estado de motivação mais relacionado ao modo como as pessoas atuam em suas tarefas laborais do que às metas e o tipo de organização em que estão inseridas (Magnan, Vasquez, Pacico, \& Hutz, 2016). A variável se difere dos níveis de satisfação do profissional com seu trabalho na medida que o engajamento trata do prazer no trabalho somado a um maior nível de ativação (i.e., vigor e absorção) (Bakker, 2011). Profissionais engajados tendem a investir mais em ações que visam seu desenvolvimento global, por vezes obtendo desempenho superior em suas atividades (Bakker, Demerouti, \& 
Sanz-Vegel, 2014). A literatura aponta como antecedentes do engajamento no trabalho o equilíbrio entre recursos pessoais (e.g., autoestima, autoeficácia, otimismo) e do trabalho (i.e., aspectos situacionais) com as demandas impostas por este (Bakker \& Demerouti, 2007; Oliveira \& Rocha, 2017).

Considerando a lacuna identificada sobre avaliação de treinamentos para uso de entrevista investigativa em casos de violência sexual, o objetivo do presente estudo foi avaliar o impacto de um programa de treinamento presencial para o uso do protocolo NICHD. Além disso, buscou-se: (1) avaliar e descrever os níveis de reação, transferência de aprendizagem, impacto do treinamento, engajamento no trabalho e autoeficácia ocupacional em intervenções com populações vulneráveis dos participantes; (2) verificar relações entre escolaridade, tempo de conclusão de curso e o tempo de atuação na avaliação de casos de VS com os índices de reação, transferência de aprendizagem, impacto do treinamento e autoeficácia ocupacional com populações em vulnerabilidade; (3) avaliar se os níveis de engajamento no trabalho atuam como antecedentes dos índices de Reação (ReARES, ReAPRO e ReaIns) e se os índices de engajamento no trabalho e índices de reação explicam os níveis da transferência de aprendizagem e impacto do treinamento no trabalho; (4) compreender quais os contextos de atuação (i.e., suporte à transferência), características do protocolo NICHD e aspectos do treinamento facilitaram/dificultaram a adesão à utilização do protocolo NICHD.

\section{Método}

Estudo misto sequencial explanatório. Tal delineamento é dividido em duas etapas distintas e interativas. Inicialmente, há a coleta e a análise da dados quantitativos (Etapa 1), em seguida, ocorre a coleta e análise de dados qualitativos (Etapa 2). Os dados qualitativos têm como objetivo auxiliar a explicar os resultados quantitativos (Creswell \& Clark, 2013).

\section{Participantes}

A amostra foi composta por 55 profissionais que realizaram o curso de formação para aplicar o NICHD. A idade média dos participantes foi 38,4 anos (DP = 8,7 anos; amplitude de 23 anos a 59 anos), sendo $85,5 \%$ mulheres. O tempo de trabalho médio dos participantes foi de 6,7 anos $(D P=5,2$ anos; amplitude de um mês a 26,6 anos) e o tempo na função foi 6,5 anos $(D P=1$ anos; amplitude de 1 mês a 26,6 anos). O nível de escolaridade foi superior completo (26\%), sendo que destes 51\% tinham ao menos um curso de especialização profissional, e 23\% eram pós-graduados (mestrado, doutorado e pós-doutorado). No total da amostra, 93\% eram profissionais de psicologia, $5 \%$ eram profissionais do direito e $2 \%$ do serviço social. A maior parte dos profissionais (64\%) referiram ter formação específica para trabalhar com crianças e adolescentes vítimas de maus-tratos. Foi observado que todos os profissionais tinham experiência no trabalho com vítimas de violência sexual. Entre esses, a média do tempo de trabalho com vítimas de VS foi 6 anos $(D P=5,9$ anos; amplitude de 1 mês a 26,6 anos).

Foram selecionados aleatoriamente seis participantes para a realização das entrevistas (Etapa 2). Destes participantes, três relataram no questionário sociodemográfico e laboral terem utilizado ao menos cinco vezes o protocolo e três relataram que não utilizaram a técnica ou não a utilizaram nos últimos dois anos. Todos os profissionais selecionados tinham formação em Psicologia, três destes cursaram pós-graduação em Psicologia (Lato Senso). A média da idade foi de 37,8 anos $(D P=9,1)$, sendo que o tempo médio de experiência foi de 5,54 anos $(D P=3,1)$. Cinco profissio- nais atuavam no serviço público (e.g., delegacias, tribunais de justiça) e um profissional em uma Organização Não Governamental (ONG).

\section{Instrumentos}

(1) Questionário sociodemográfico e laboral. Tem como objetivo avaliar características sociodemográficas e laborais dos participantes (e.g., formação acadêmica, experiência na avaliação de vítimas de violência sexual contra crianças e adolescentes).

(2) Questionário de Avaliação de Reações. O questionário é dividido em duas subescalas, totalizando 39 itens (Abbad, 1999). A primeira subescala é a Escala de Reação ao Curso (ERC) e tem por objetivo mensurar a satisfação dos participantes em relação à qualidade do curso. O constructo é divido em dois fatores correlacionados entre si. O primeiro (F1) é chamado de Reação à Programação e ao Apoio (ReaPRO) O segundo fator (F2), é chamado de Reações aos resultados, aplicabilidade e expectativas de suporte (ReaRES). Ambos os fatores apresentaram consistências internas satisfatórias 0,89 e 0,95, respectivamente (Abbad, 1999). A segunda subescala é a Escala de Reação ao Desempenho do Instrutor (ReaIns) que tem por objetivo mensurar a satisfação dos participantes em relação ao desempenho do instrutor. Tal escala apresentou uma consistência interna satisfatória $(\alpha=0,96)$ (Abbad, Zerbini, \& Borges-Ferreira, 2012).

(3) Escala de Transferência de Aprendizagem (ETA). Tem como objetivo averiguar os níveis de transferência de aprendizagem dos conteúdos abordados no treinamento ao contexto de trabalho dos profissionais treinados. Trata-se de uma escala com 17 itens e cinco possibilidades de resposta. A escala foi desenvolvida com base no instrumento proposto por Freitas (2013), em uma pesquisa que avaliou os índices de transferência de aprendizagem de uma tecnologia social para profissionais de psicologia que trabalham com vítimas de violência sexual contra crianças e adolescentes.

(4) Escala de Auto Avaliação de Impacto do Treinamento no Trabalho - medida em amplitude (EAITT). Trata-se de uma escala desenvolvida e validada por Abbad (1999) e adaptada por Abbad, Pilati, Borges-Andrade e Sallorenzo (2012) com o objetivo de mensurar a auto avaliação do participante no que tange aos efeitos produzidos pelo treinamento em seu desempenho, motivação, autoconfiança e abertura do participante a mudanças nos processos de trabalho. O instrumento é composto de 12 itens associados a cinco possibilidades de respostas. A escala é composta por dois fatores chamados de impacto do treinamento no desempenho e impacto de treinamento nas atitudes e apresenta consistência interna satisfatória $(\alpha=0,86)$ (Abbad et al., 2012).

(5) Escala Utrecht de Engajamento no Trabalho (UWES). A escala foi desenvolvida por Schaufeli, Salanova, González-Romá e Bakker (2002) e adaptada por Vazquez et al. (2015). É composta por 17 itens que avaliam o engajamento no trabalho. A análise dos resultados se dá pela soma dos itens na escala likert e os resultados são transformados em percentis conforme normas brasileiras (Vazquez et al., 2015). A consistência interna da escala é de $\alpha=0.95$.

(6) Escala de Autoeficácia Ocupacional em Intervenções com Populações Vulneráveis (EAO-IPV). Esta escala tem como objetivo investigar a autoeficácia que os profissionais possuem sobre suas habilidades em trabalhar com populações em situação de vulnerabilidade, avaliando aspectos relacionados a direitos humanos, condutas de paz e mediação de conflitos. A escala possui 18 itens variando de 1 (discordo totalmente) a 5 (concordo totalmente). Foi observado que a escala apresentou consistência interna satisfatória $(\alpha=0,86)$ (Freitas et al., 2017). 
(7) Entrevista semiestruturada. O objetivo de tal entrevista foi compreender quais os contextos de atuação e características do protocolo NICHD facilitaram/dificultaram sua utilização na prática profissional. As perguntas norteadoras da entrevista foram: (1) Me conta sobre como foi a experiência de ter realizado o curso sobre o protocolo NICHD; (2) O que te motivou a fazer esse curso?; (3) As tuas expectativas foram atendidas?; (4) Quais os aspectos positivos você destacaria no curso que você fez; (5) Quais os aspectos você considera que devam ser melhorados? (6) Você colocou em prática conteúdos aprendidos nesse curso? Quais?; (7) Você já aplicou o protocolo NICHD? Me conta como foi essa experiência; (8) Que aspectos relacionados à instituição em que você trabalha você considera facilitar ou dificultar o uso do protocolo; (9) Nosso objetivo é melhorar o treinamento para o uso do protocolo NICHD. Tem algo a mais sobre o curso que você gostaria de comentar?

\section{Procedimentos de Coleta de Dados e Cuidados Éticos}

O presente estudo foi aprovado pelo Comitê de Ética em Pesquisa da Pontifícia Universidade Católica do Rio Grande do Sul, sob parecer n 2.353.659. Todos os participantes foram informados sobre a natureza do estudo e seus objetivos, sendo assegurado o sigilo da identidade dos mesmos. Os procedimentos éticos estão de acordo com a resolução 510/16 do Conselho Nacional de Saúde (2016).

Na Etapa 1, quantitativa, todos os profissionais que participaram de treinamentos para a utilização do protocolo NICHD entre 2015 e 2017 foram convidados via e-mail a responder ao formulário on-line (via Qualtrics) com as escalas para avaliação do treinamento. $\mathrm{Na}$ Etapa 2, qualitativa, seis destes profissionais foram selecionados com base na frequência de utilização do protocolo NICHD em suas práticas profissionais (i.e., três profissionais que utilizaram pelo menos cinco vezes o protocolo e três profissionais que não utilizaram a técnica ou não a utilizaram nos últimos dois anos). As entrevistas foram conduzidas por duas pesquisadoras do grupo de pesquisa que não estavam vinculadas ao treinamento. As entrevistas foram gravadas em áudio e transcritas para posterior análise. Tendo em vista que os participantes realizaram o treinamento entre 2015 e 2017, houve na ocasião a cobrança pelo treinamento. Possíveis vieses foram reduzidos por meio da coleta online sem a identificação dos participantes para que estes pudessem avaliar livremente o treinamento e instrutor.

O modelo de treinamento adotado foi desenvolvido com base nos estudos de Aznar-Blefari e Padilha (2015), adaptado a um período de $20 \mathrm{~h}$ de instrução, tendo em vista a realidade brasileira na qual muitas instituições não permitem que seus colaboradores utilizem os horários de trabalho para treinamento (baixo suporte à transferência). As $20 \mathrm{~h}$ foram distribuídas em dois dias (i.e., geralmente um final de semana) e o conteúdo foi dividido em quatro módulos: (1) Apresentação da proposta do treinamento, definições, consequências e principais dinâmicas dos atos abusivos; (2) Boas-práticas em entrevistas investigativas (e.g., perguntas adequadas, formatação do setting de entrevista, uso de artefatos visuais, postura profissional) e discussão de estudos sobre desenvolvimento infantil que validam a utilização do protocolo NICHD; (3) Etapas da entrevista foram estudadas com exemplos simulados e os participantes realizaram role-playing de entrevistas investigativas; (4) Simulação de entrevistas sob supervisão do instrutor em pequenos grupos de no máximo três participantes. Tendo em vista a importância da supervisão de casos ou revisão de pares das entrevistas após a realização do treinamento (AznarBlefari \& Padilha, 2015; Lamb, 2016), para cada turma foi criado um grupo de WhatsApp para facilitar o contato entre os alunos e com o instrutor.

\section{Procedimentos de Análise de Dados}

Para responder ao objetivo específico 1 as médias, desvios-padrão e amplitudes das escalas foram mensuradas. A fim de calcular as estatísticas descritivas das variáveis investigadas foram utilizados os escores brutos para cada variável. O escore bruto de cada variável foi obtido pela soma dos itens da escala, dividido pelo número de itens da respectiva dimensão, de modo a ser obtida a média ponderada dessa variável.

Para atender ao objetivo específico 2, inicialmente a normalidade das variáveis foi testada por meio dos testes Kolmogorov-Smirnov e Shapiro-Wilk. Como a maior parte das variáveis não apresentou uma distribuição normal, optou-se pela análise de correlação de Spearman (rho) e o cálculo do intervalo de confiança. Com respeito ao objetivo específico 3, foi realizada uma análise de regressão linear para cada variável desfecho (Reação à Programação e ao Apoio; Reação aos Resultados, Aplicabilidade e Expectativas de Suporte e Reação ao Desempenho do Intrutor; Impacto do Treinamento no Trabalho; e Transferência de Aprendizagem), sendo que variáveis independentes foram definidas com base nos resultados da análise de correlação (objetivo 2). Desta forma foi investigado se os níveis de engajamento no trabalho atuam como antecedentes dos índices de Reação (Reação à Programação e ao Apoio; Reação aos Resultados, Aplicabilidade e Expectativas de Suporte e Reação ao Desempenho do Instrutor). Além disso, foi avaliado se índices de engajamento no trabalho e índices de Reação explicam os níveis da Transferência de Aprendizagem e Impacto do Treinamento no Trabalho. A análise de regressão linear foi realizada utilizando o método Enter com bootstrap (1000 amostras), devido à ausência de normalidade das variáveis (Field, 2005) e para ampliar a robustez dos resultados, tendo em vista o tamanho da amostra.

Para uma melhor compreensão dos contextos de atuação, as características do protocolo NICHD e os aspectos do treinamento que facilitaram/dificultaram a adesão ao uso da técnica de entrevista pelos profissionais em suas atuações (objetivo específico 4), as entrevistas foram analisadas por meio do método Análise Temática (Braun \& Clarke, 2006). Inicialmente as entrevistas realizadas foram transcritas e revisadas seguindo os seguintes passos: (1) Familiarização com dados; (2) Produção de códigos; (3) Busca de temas; (4) Revisão dos temas; (5) Definição e nomeação dos temas; e (6) Produção do relatório (Braun \& Clarke, 2006; Souza, 2019). Por meio de uma lógica indutiva, dados brutos sem uma predefinição de categorias foram identificados por dois juízes independentes que discutiram os achados chegando a um consenso com auxílio de um terceiro juiz.

\section{Resultados}

A seção de resultados está organizada em duas partes. A primeira apresenta os resultados das análises quantitativas dos dados. A segunda parte apresenta a análise qualitativa das entrevistas realizadas.

\section{Etapa 1 - Avaliação de Impacto do Treinamento (Quantita- tiva)}

A avaliação e descrição (objetivo 1) dos níveis de reação, transferência de aprendizagem, impacto do treinamento, engajamento no trabalho e autoeficácia ocupacional em intervenções com populações vulneráveis, as médias, desvios-padrão e amplitudes estão apresentadas na Tabela 1. Foi possível observar que 
os profissionais apresentaram altos índices de autoeficácia ocupacional em intervenções com populações vulneráveis (AOIPV) e engajamento no trabalho. Os níveis de reação ao treinamento (Reação à programação e ao apoio - ReaPRO; Reação aos resultados, aplicabilidade e expectativas de suporte - ReaRES e; Reação ao desempenho do intrutor - ReaIns) apresentaram resultados médio-altos. Por fim, os efeitos do treinamento no trabalho dos profissionais (Transferência de Aprendizagem - ETA e Impacto do Treinamento no Trabalho) indicaram resultados medianos e valores médio-altos, respectivamente.

Em relação ao objetivo 2, que buscou avaliar a hipótese de que

Tabela 1

Médias, desvios-padrão e amplitudes das variáveis analisadas neste estudo

\begin{tabular}{ccccc}
\hline \multirow{2}{*}{ Instrumento } & Média & $\begin{array}{c}\text { Desvio- } \\
\text { Padrão }\end{array}$ & \multicolumn{2}{c}{ Amplitude } \\
\cline { 5 - 6 } & & & Mínimo & Máximo \\
\hline ReaPRO & 4,3 & 0,6 & 2,5 & 5,0 \\
ReaRES & 4,2 & 0,5 & 2,9 & 5,0 \\
ReaIns & 4,5 & 0,5 & 2,8 & 5,0 \\
TA & 4,0 & 0,5 & 2,9 & 5,0 \\
Impacto & 4,4 & 0,4 & 3,3 & 5,0 \\
AOIPV & 4,2 & 0,6 & 1,6 & 5,0 \\
Engajamento no Trabalho & 5,7 & 0,8 & 3,5 & 6,9 \\
\hline
\end{tabular}

Nota. Medidas de Reação - Reação à Programação (ReaPRO), Reação aos Resultados, Aplicabilidade e Expectativas de Suporte (ReaRES) e, Reação ao Desempenho do Instrutor (ReaIns). Medidas de efeito do treinamento no trabalho - Transferência de Aprendizagem (TA) e, Impacto. Autoeficácia ocupacional em intervenções com populações vulneráveis (AOIPV). haveria relações entre a escolaridade, tempo de conclusão de curso e o tempo de atuação na avaliação de casos de VS com os índices de reação, transferência de aprendizagem, impacto do treinamento e AOIPV, foi observado que a escolaridade, o tempo de trabalho e a autoeficácia ocupacional com populações em vulnerabilidade não apresentaram relações significativas com as demais variáveis investigadas. Os índices de engajamento no trabalho apresentaram associações positivas com os níveis de ReaPRO, transferência de aprendizagem e impacto do treinamento no trabalho.

Os índices de Reação (i.e., ReaPRO, ReaRES, ReaIns) se correlacionaram positivamente entre si e com as variáveis impacto do treinamento no trabalho e transferência de aprendizagem. Por fim, a transferência de aprendizagem esteve positivamente associada ao impacto do treinamento no trabalho (Tabela 2).

$\mathrm{Na}$ regressão (objetivo 3) foi investigado se as dimensões associadas ao treinamento (i.e., Reação) seriam explicadas pelo engajamento no trabalho (Tabela 3). Foi possível observar que o engajamento no trabalho atuou como um preditor dos níveis de reação à programação e ao apoio $(B=0,32 ; p<0,05)$ explicando $9 \%$ da variância (Bootstrap 0,04 a 0,40 ) (Tabela 3).

Com o objetivo de aprofundar a compreensão dos resultados observados na análise de correlação, foi investigado se os índices de transferência de aprendizagem e impacto do treinamento seriam explicados pelos níveis de Engajamento no trabalho e índices de Reação. Os resultados apontaram que o engajamento no trabalho e o fator reação aos resultados, aplicabilidade e expectativas de suporte (ReaRES) colaboram para explicar 37\% da variância da transferência de aprendizagem e do impacto do treinamento no

Tabela 2

Resultados da análise de correlações entre Escolaridade, Tempo de Trabalho com Vitimas de VS, Autoeficácia Ocupacional em Intervenções com Populações Vulneráveis, Engajamento no

Trabalho, Reação à Programação e ao Apoio, Reação aos Resultados, Aplicabilidade e Expectativas de Suporte, Reação ao Desempenho do Tutor, Transferência de Aprendizagem e Impacto do Treinamento no Trabalho

\begin{tabular}{|c|c|c|c|c|c|c|c|c|}
\hline Variáveis & 1 & 2 & 3 & 4 & 5 & 6 & 7 & 8 \\
\hline \multicolumn{9}{|l|}{$1 \mathrm{Esc}$} \\
\hline \multirow{2}{*}{$2 \mathrm{TT}$} & $-0,05$ & & & & & & & \\
\hline & {$[-0,31-0,21]$} & & & & & & & \\
\hline \multirow{2}{*}{3 AOIPV } & $-0,16$ & 0,14 & & & & & & \\
\hline & {$[-0,41-0,11]$} & {$[-0,13-0,39]$} & & & & & & \\
\hline \multirow{2}{*}{4 ET } & $-0,12$ & $-0,14$ & 0,26 & & & & & \\
\hline & {$[-0,37-0,15]$} & {$[-0,39-0,13]$} & {$[-0,00-0,49]$} & & & & & \\
\hline \multirow{2}{*}{5 ReaPRO } & $-0,14$ & $-0,05$ & 0,25 & $0,32^{*}$ & & & & \\
\hline & {$[-0,39-0,13]$} & {$[-0,31-0,22]$} & {$[-0,02-0,48]$} & {$[0,06-0,54]$} & & & & \\
\hline \multirow{2}{*}{6 ReaRES } & $-0,01$ & $-0,08$ & 0,20 & 0,19 & $0,62^{*}$ & & & \\
\hline & {$[-0,27-0,26]$} & {$[-0,34-0,19]$} & {$[-0,07-0,44]$} & {$[-0,08-0,43]$} & {$[0,43-0,76]$} & & & \\
\hline \multirow{2}{*}{7 ReaIns } & $-0,04$ & 0,01 & 0,16 & 0,20 & $0,78^{*}$ & $0,58^{*}$ & & \\
\hline & {$[-0,30-0,23]$} & {$[-0,26-0,27]$} & {$[-0,11-0,41]$} & {$[-0,07-0,44]$} & {$[0,64-0,86]$} & {$[0,37-0,73]$} & & \\
\hline \multirow{2}{*}{8 ITT } & 0,01 & $-0,25$ & 0,25 & $0,38^{*}$ & $0,39 *$ & $0,57^{*}$ & $0,42^{*}$ & \\
\hline & {$[-0,26-0,27]$} & {$[-0,48-0,01]$} & {$[-0,01-0,49]$} & {$[0,13-0,59]$} & {$[0,14-0,60]$} & {$[0,36-0,72]$} & {$[0,17-0,61]$} & \\
\hline \multirow{2}{*}{$9 \mathrm{TA}$} & 0,03 & $-0,26$ & 0,25 & $0,28^{*}$ & $0,38^{*}$ & $0,64^{*}$ & $0,46^{*}$ & $0,75^{*}$ \\
\hline & {$[-0,24-0,29]$} & {$[-0,49-0,01]$} & {$[-0,01-0,48]$} & {$[0,02-0,51]$} & {$[0,13-0,59]$} & {$[0,46-0,78]$} & {$[0,22-0,65]$} & {$[0,60-0,85]$} \\
\hline
\end{tabular}

Nota. $*=p<0,05 ;$ Esc = Escolaridade; TT = Tempo de trabalho: específico com Vítimas de VS; AOIPV = Autoeficácia Ocupacional em Intervenções com Populações Vulneráveis; ET = Engajamento no trabalho; Índices de Reação (ReaPRO = Reação à Programação e ao Apoio; ReaRES = Reação aos Resultados, Aplicabilidade e Expectativas de Suporte; ReaIns = Reação ao Desempenho do Instrutor). Medidas de efeito do treinamento no trabalho (ITT= Impacto do Treinamento no Trabalho; TA = Transferência de Aprendizagem). Os valores entre colchetes indicam o intervalo de confiança de $95 \%$ para cada correlação.

Tabela 3

Contribuição do Engajamento no Trabalbo sobre os niveis de Reação à Programação e ao Apoio, Reação aos Resultados, Aplicabilidade e Expectativas de Suporte e Reação ao Desempenho do Instrutor.

\begin{tabular}{|c|c|c|c|c|c|c|c|c|c|}
\hline \multirow{3}{*}{$\mathrm{P}$} & \multicolumn{3}{|c|}{ ReaPRO } & \multicolumn{3}{|c|}{ ReaRES } & \multicolumn{3}{|c|}{ ReaIns } \\
\hline & \multicolumn{3}{|c|}{$R^{2} A d j=0,09$} & \multicolumn{3}{|c|}{$R^{2} A d j=0,02$} & \multicolumn{3}{|c|}{$\mathrm{R}^{2} \mathrm{Adj}=0,02$} \\
\hline & $R^{2}$ & $\beta$ & Bootstrap (I.C. 95\%) & $R^{2}$ & $B$ & Bootstrap (I.C. 95\%) & $R^{2}$ & $B$ & Bootstrap (I.C. 95\%) \\
\hline Eng & 0,10 & $0,32 *$ & 0,04 a 0,40 & 0,03 & 0,19 & $-0,06$ a 0,33 & 0,04 & 0,20 & $-0,06$ a 0,32 \\
\hline
\end{tabular}

Nota. $*=p<0,05 ; \mathrm{P}=$ Preditor; Eng = Engajamento; ReaPRO = Reação à Programação e ao Apoio; ReaRES = Reação aos Resultados, Aplicabilidade e Expectativas de Suporte; ReaIns

= Escala de Reação ao Desempenho do Instrutor. 
Tabela 4

Contribuição das variáveis Engajamento no Trabalho, Reação à Programação e ao Apoio, Reação aos Resultados, Aplicabilidade e Expectativas de Suporte, Reação ao Desempenho do Instrutor sobre os niveis de Transferência de Aprendizagem e Impacto do Treinamento no Trabalho.

\begin{tabular}{|c|c|c|c|c|c|c|}
\hline \multirow{4}{*}{$\mathrm{P}$} & \multicolumn{3}{|c|}{ TA } & \multicolumn{3}{|c|}{ ITT } \\
\hline & \multicolumn{3}{|c|}{$\mathrm{R}^{2} \mathrm{Adj}=0,37$} & \multicolumn{3}{|c|}{$\mathrm{R}^{2} \mathrm{Adj}=0,37$} \\
\hline & $R^{2}$ & $\beta$ & $\begin{array}{l}\text { Bootstrap } \\
\text { (I.C. } 95 \% \text { ) }\end{array}$ & $R^{2}$ & $\beta$ & $\begin{array}{l}\text { Bootstrap } \\
\text { (I.C. } 95 \% \text { ) }\end{array}$ \\
\hline & 0,42 & & & 0,42 & & \\
\hline Eng & & $0,31^{*}$ & 0,05 a 0,37 & & $0,31 *$ & 0,06 a 0,37 \\
\hline $\begin{array}{l}\text { Rea- } \\
\text { PRO }\end{array}$ & & $-0,187$ & $-0,38$ a 0,11 & & $-0,17$ & - 0,39 a 0,13 \\
\hline ReaRES & & $0,51^{*}$ & 0,22 a 0,71 & & $0,51^{*}$ & 0,22 a 0,70 \\
\hline ReaIns & & 0,19 & $-0,09$ a 0,51 & & 0,19 & $-0,10$ a 0,50 \\
\hline
\end{tabular}

Nota. $*=p<0,05 ; \mathrm{P}=$ Preditores; Eng = Engajamento no Trabalho; Níveis de Reação (RePPRO = Reação à Programação e ao Apoio; ReaRES = Reação aos Resultados, Aplicabilidade e Expectativas de Suporte; ReaIns = Reação ao Desempenho do Instrutor); Medidas de efeito do treinamento no trabalho (TA $=$ Transferência de Aprendizagem; ITT $=$ Impacto do Treinamento no Trabalho)

\section{Etapa 2 - Avaliação Qualitativa do Treinamento e Condições Para Aplicabilidade do Conteúdo}

Com o objetivo de compreender quais os contextos de atuação, as características do protocolo NICHD e os aspectos do treinamento que facilitaram/dificultaram a adesão do uso da técnica foram realizadas entrevistas com seis profissionais que participaram da Etapa 1 do estudo, sendo um homem e cinco mulheres. $\mathrm{O}$ corpus de análise foi composto por um total de 226 minutos e 09 segundos. O áudio foi transcrito em Times New Roman (tamanho 12, espaçamento $1,5 \mathrm{~cm})$ em 87 páginas $(M=14,5 ; D P=5,28)$ e, 28.166 palavras $(M=4694,33 ; D P=2057,73)$. A Análise Temática foi conduzida por meio de uma lógica dedutiva, na qual foram utilizados recursos conceituais e teóricos para analisar os dados. Os dados foram analisados a partir das variáveis reação, engajamento e suporte. A escolha dos temas ocorreu por similaridade (de Farias, Dutra-Thomé, Koller, \& Castro, 2020) e teve como questões norteadoras (1) Quais os contextos de atuação (i.e., suporte à transferência) dos egressos? e; (2) Quais características do protocolo NICHD e aspectos do treinamento facilitaram/dificultaram a adesão à utilização do protocolo NICHD. A análise resultou em seis temas e 24 subtemas.

O primeiro tema aborda os aspectos positivos do treinamento (1). Tal tema refere-se à reação dos participantes ao treinamento, aspecto avaliado também por autorrelato na etapa 1 do estudo. $O$ segundo tema aborda os aspectos que requerem aperfeiçoamento (2). Os participantes apresentaram queixas e aspectos que os desagradaram no curso. Trata-se de pontos que necessitam atenção pois podem prejudicar os níveis de reação ao treinamento.

A motivação e expectativas (3) quanto ao treinamento apresenta como os participantes desenvolveram o interesse por se capacitar na área ou entraram em contato com a proposta de treinamento. No tema aplicações (4), são apresentados exemplos de utilização e a opinião sobre dos participantes sobre os tópicos estudados no treinamento. O tema suporte à transferência (5) demonstra a colaboração ou falta de apoio das instituições de proteção em que os profissionais atuam à adoção de novas práticas em entrevista investigativa. Por fim, no tema Sugestões (6) os participantes apresentam conteúdos a serem incluídos e suporte para futuras turmas de treinamento (Tabela 5).
Um dos objetivos deste estudo foi avaliar e descrever os níveis de reação, transferência de aprendizagem, impacto do treinamento, engajamento no trabalho e autoeficácia ocupacional em intervenções com populações vulneráveis dos participantes. Os achados sugerem que os profissionais que participaram do treinamento avaliaram de forma positiva as suas habilidades no trabalho, estão envolvidos com o seu trabalho, satisfeitos com o treinamento e perceberam impacto positivo na sua prática profissional. Tais evidências podem contribuir para qualificar a atuação destes profissionais na condução de avaliações de crianças e adolescentes vítimas de violência sexual. A literatura demonstra que os níveis de impacto do treinamento referem-se aos benefícios da capacitação para além daqueles previstos no programa de treinamento (impacto em amplitude) (Abbad, Gama, \& Borges-Andrade, 2000; Schoeb et al., 2019) e estão relacionados positivamente à apreensão dos conhecimentos e habilidades, ao armazenamento das informações e à capacidade de utilizar o conteúdo aprendido em situações distintas (Pilati \& Abbad, 2005).

Os níveis elevados de autoeficácia ocupacional em intervenções com populações vulneráveis e o engajamento no trabalho sinalizam que os profissionais participantes do treinamento apresentam fatores protetivos à saúde do trabalhador (e.g., redução de afetos negativos e burnout) e podem potencializar a transferência do aprendizado (Freitas, Silva, Damásio, Koller, \& Teixeira, 2014; Schaufeli, 2014). Sendo assim, a utilização de um novo recurso (i.e., protocolo NICHD) pode contribuir como fator protetivo ao estresse laboral (i.e., demandas) e estes achados podem sugerir uma postura mais motivada e segura do participante quanto à utilização do protocolo NICHD. Tais resultados podem ser interpretados à luz das entrevistas realizadas na Etapa 2 deste estudo. Foi possível identificar que alguns participantes compreendem o protocolo NICHD como uma ferramenta que contribui ao desenvolvimento do trabalho devido ao melhor estabelecimento de rapport, uso de questões abertas e, por se tratar de uma técnica que facilita a revelação por parte da criança. Tal percepção dos participantes condiz com os achados na literatura que demonstram a efetividade do uso do Protocolo NICHD na avaliação de crianças e adolescentes vítimas de violência (Faller, 2015, Lamb et al., 2008; Lamb, 2016; La Rooy et al., 2015).

Quanto ao objetivo 2 e 3, este estudo não encontrou relações entre os níveis de escolaridade, tempo de conclusão do curso e o tempo de atuação na avaliação de casos de VS com os índices de reação, transferência de aprendizagem, impacto do treinamento e autoeficácia ocupacional. Os resultados de pesquisas sobre a relação entre as medidas de impacto do treinamento no trabalho não são consistentes. Como exemplo, um estudo nacional que objetivou avaliar medidas de reação, aprendizagem e impacto em 211 profissionais egressos de três treinamentos em uma Agência Reguladora, não identificou correlação entre aprendizagem e impacto, mas, observou relação significativa entre reação e impacto. Há estudos em que a correlação da aprendizagem com o impacto do treinamento existe de maneira fraca e outros que demonstram ser significativa a relação (referências destes estudos). Tais diferenças entre resultados são causadas por inúmeros fatores como diferenças nas medidas de aprendizagem (e.g., medidas de autorrelato ou prova situacional) (Araújo, Abbad, \& Freitas, 2019).

Foi possível identificar que os níveis de engajamento no trabalho predizem a reação. E quando somados antecedem a transferência de aprendizagem e o impacto do treinamento (i.e., transferência de treinamento). Os níveis de reação se relacionam positivamente com o engajamento no trabalho e negativamente com a intenção de rotatividade. O engajamento no trabalho é me- 
Tabela 5

Temas, subtemas e unidades de análise representativas

\begin{tabular}{|c|c|c|}
\hline Temas & Subtemas & Unidades Representativas \\
\hline \multirow{5}{*}{$\begin{array}{l}\text { Aspectos positivos } \\
\quad \text { (Reação) }\end{array}$} & Prática (role-playing) & $\begin{array}{l}\text { E2 "A teoria foi bem bacana mais a parte prática, quando se coloca tem aquele momento frente a frente que o } \\
\text { professor vai instrumentalizando, esse momento é bem essencial. (...) Porque realmente mostra o que vai ser." }\end{array}$ \\
\hline & Experiência do instrutor & E3 "Ele trouxe muito da experiência dele né. Isso me chamou atenção. Acho que foi bem satisfatório." \\
\hline & Fundamentação teórica & $\begin{array}{c}\text { E4 "em momento algum o professor fez partindo de um senso comum ou de uma experiência. Acho que ele } \\
\text { veio muito bem fundamentado teoricamente." }\end{array}$ \\
\hline & Materiais e infraestrutura & $\begin{array}{l}\text { E2 "A Parte audiovisual que ele utilizou no curso ne. O vídeo comparando a entrevista. Também mostrou uma } \\
\text { entrevista ruim ne. A concentração da criança. A Tensão dela né. Principalmente na questão do espaço na sala } \\
\text { pra não ter muita coisa que chame atenção. (...) Esse foi um momento bem importante" }\end{array}$ \\
\hline & $\begin{array}{l}\text { Interação com os } \\
\text { participantes }\end{array}$ & $\begin{array}{c}\text { E6 "A troca de experiência por ter profissionais de outras áreas. A maioria eram psicólogos. (...) eram de } \\
\text { ambientes diferentes (...) troca de experiências sobre os municípios”. }\end{array}$ \\
\hline
\end{tabular}

E1 "Às vezes eu tive alguma dúvida assim, que eu entrava, mandava e-mail e tudo e às vezes não obtinha resposta né. Aí é um pouco de organização nesse sentido".

Aspectos que requerem aperfeiçoamento

(Reação)

Preocupação com a exposição dos participantes

Grupo do WhatsApp

Publicação científica

Treinamento como postura

Motivação e ética

expectativas

(Engajamento)

Recursos técnicos para obtenção de relato

Uso de Questões abertas

Rapport

Segurança na entrevista

Gravação como prova

Aplicações (Transferência de Aprendizagem)

Necessidade de treinamento e supervisão

Extensão do Protocolo NICHD

Maior facilidade na obtenção do relato

Avaliação Criminal

Apoio

Suporte à transferência Sobrecarga de trabalho

Precariedade do local de trabalho

Financiamento do treinamento

Treinamento continuado

Sugestões

Crianças e adolescentes com deficiência
E4 "Eu nunca gostei de me expor em público. Acho que poderia utilizar filmes que eventualmente abordam uma entrevista. Acho que no meu ponto de vista é um pouco mais válido e não expõe tanto os participantes".

E6 "foi criado para a gente tirar dúvidas referentes ao nosso uso, do inquérito tudo. E na verdade o grupo é assim bem parado né. (...) a ideia é bem legal mas se esse grupo funcionasse de fato sabe (...) A gente teve o curso e trocou bastante experiência, mas na prática você precisava desse respaldo. "

E1 "Eu comecei a pesquisar recursos para melhorar essa prática, foi aí que eu descobri o protocolo. Eu não sabia que existia. (...) Existe um artigo né, acho que de treze páginas assim muito bom. Esse artigo me convenceu."

E4 "A gente vê a quantidade de entrevistas mal feitas pelos relatórios que chegam aqui pra nós, ou mesmo pelas reportagens dos juízes. (...). Daí eu comecei a impor essa obrigação quase ética de quando for psicóloga que atua em casos envolvendo esse tipo de situação de que eu deveria ter o mínimo de capacitação técnica, de qualificação para abordar adequadamente uma criança. "

E6 "Eu já estava com interesse de procurar um método quando eu trabalhava na secretaria da assistência social, a gente não tinha psicóloga dentro do fórum e ficava por minha responsabilidade. Eu percebia que tinham muitas falhas por falta do método. (...) Eu sentia muita falta assim porque eu percebia que chegava num momento e a criança não falava mais."

E1 "Eu tinha uma tendência de fazer uma entrevista mais rápida (...) então com o protocolo eu me policio para fazer bastantes perguntas abertas. É o diferencial dele."

E5 “tem o rapport isso traz uma tranquilidade. Eu já participei de outras audiências. Que as crianças falavam por exemplo junto com o juiz o promotor e o advogado. Era super constrangedor"

E5 "usar o protocolo te da uma segurança maior. Durante a entrevista. No caso do depoimento especial a gente está fazendo uma coleta de depoimento"

E1 "Então, apesar de ser algo um pouco polêmico ainda, eu não vi nada grave que possa, uma vez que tenha autorização do responsável da criança. Então desde o início eu fiz um formulário que é preenchido na hora com o nome da criança, nome do responsável, a data e eu peço que o responsável leia o formulário e assine se ele concordar"

E3 “a gente vai pro abrigo. Lá que se faz a escuta especial né. Então eu vejo o tempo todo o pessoal falando do protocolo e eu tenho formação e estudei o protocolo. Fez o curso com quem? Quanto tempo de curso? Faz supervisão com quem? A gente não pode usar um instrumento que tu não está habilitado em horas e não buscou supervisão. Vai aplicar errado."

E4 "Achei o protocolo muito válido, muito importante. Mas em termos de como que era, acho que me marcou foi isso assim. De sentir que era muito longo, muito cansativo, tanto pra mim, quanto pra criança”.

E6 "na experiência foi que a criança fala mesmo. Ela conta o que aconteceu. (...) De fato o relato da criança é um pouquinho maior."

E4 "Na vara de família não é necessariamente o momento de aprofundar e aplicar o protocolo (...) Com o tempo assim a gente foi deixando essa função ne para vara criminal"

E4 “(...)a gente tem autonomia para escolher os métodos, instrumentos que a gente vai utilizar no nosso trabalho"

E4 “(...) a demanda de serviços poderia ser considerada uma das dificuldades (...) o sistema judiciário brasileiro está abarrotado de processos. Não me permite fazer perícias tão aprofundadas”

E1 "A barreira que encontro é a questão de instalações físicas (...) a minha sala está em um ponto inadequado entre a cela e a cozinha (...) eventualmente algum preso fica berrando, chutando as grades da cela"

E4 “(...) eu priorizo muito o conhecimento teórico técnico. Busco muito isso. Daí eu fui lá, busquei o curso e paguei por isso. Não foi o tribunal que pagou (...) o tribunal disponibilizou a sala"

E4 "O professor se mostrou super à disposição pra nós fazer tipo uma consultoria com os casos que a gente aplicasse a entrevista e infelizmente ele não teve disponibilidade para dar conta do que ele se propôs. ”

E1 “Crianças com deficiência cognitiva ou pouco estimuladas. Que criança pouco estimulada tem um desenvolvimento atrasado né. Ou uma criança de oito anos que não é alfabetizada, ou até com mais idade. Então realmente o protocolo como ele está confunde a criança. Teria que ser um protocolo mais enxuto. Já fiz a entrevista com criança com deficiência auditiva também." 
diador da relação entre a reação com o treinamento e a intenção de rotatividade. Ou seja, quanto maior a reação com o treinamento e o engajamento no trabalho menor a chance de profissionais manifestarem intenção em sair da organização (Memon, Salleh, \& Baharom, 2016).

De forma semelhante, um estudo objetivou comparar níveis de engajamento no trabalho e pessoal com a percepção do treinamento e impacto no trabalho. Para tanto foram avaliados 304 trabalhadores em tempo integral no Reino Unido. Conforme previsto, o engajamento no trabalho mediou a relação entre percepções do treinamento e a proatividade da tarefa em um grau semelhante (Fletcher, 2015).

Outro resultado observado foi que os fatores de reação ao treinamento (i.e., Reação à Programação e ao Apoio; Reação aos Resultados, Aplicabilidade e Expectativas de Suporte; Reação ao Desempenho do Instrutor) estão relacionados positivamente, o que era esperado tendo em vista o constructo avaliado (Abbad, 1999; Abbad, Zerbini, \& Borges-Ferreira, 2012). Da mesma forma, é possível observar que tais fatores de reação ao treinamento estiveram relacionados ao impacto do treinamento no trabalho e à transferência de aprendizagem, em outras palavras, quanto mais satisfeito o participante se sentiu com o treinamento para o uso do Protocolo NICHD, maior a percepção dele sobre as consequências imediatas no seu desempenho laboral, motivação, autoconfiança e maior a aplicação dos conhecimentos aprendidos. A relação entre as variáveis de qualidade do treinamento (i.e., reação, transferência de aprendizagem e impacto do treinamento no trabalho) já havia sido observada na literatura e indica que o treinamento gera mudanças não somente nos conhecimentos e habilidades treinadas mas em uma qualificação global de condutas laborais (Abbad, Gama, \& Borges-Andrade, 2000; Homklin, Takashi, \& Techakanont, 2013; Tamayo \& Abbad, 2006).

Um estudo objetivou investigar o relacionamento entre o autoconceito profissional, suporte à transferência e impacto do treinamento no trabalho em profissionais da INFRAERO e do Correios (ECT). Os achados apontaram direção semelhante ao observado nesta pesquisa, quanto maior a percepção de suporte/ reação maior a percepção de impacto do treinamento no trabalho (Tamayo \& Abbad, 2006).

Por mais que se evidencie a importância da expectativa dos participantes sobre a aplicabilidade do treinamento e do suporte à transferência, tal realidade não é constante entre os participantes. $\mathrm{Na}$ análise das entrevistas, o tema "suporte à aprendizagem" sinaliza a precariedade do local de trabalho de alguns profissionais e a falta de financiamento do curso. Da mesma forma, o programa de treinamento desenvolvido e avaliado neste estudo precisou ser adaptado a $20 \mathrm{~h}$ de atividades em finais de semana por solicitação dos participantes que não receberam autorização de seus locais de trabalho (públicos ou privados) para a realização a capacitação em horário comercial. Há um consenso na literatura quanto à relação entre o suporte à transferência e o impacto do treinamento no trabalho (Abbad, 1999; Abbad et al., 2000; Bell et al., 2017).

Um recente estudo teve como objetivo avaliar a influência de percepções de suporte sobre impacto do treinamento no trabalho em 2.975 egressos de três treinamentos oferecidos pela Universidade Corporativa de uma empresa pública. Os resultados apontam que o suporte à transferência é preditor do impacto do treinamento no trabalho e que a força motivacional é mediadora entre estas variáveis (Moreira, Abbad, Cualheta, \& Neiva, 2019)

Para que a Lei 13.431 (Brasil, 2017) possa ser cumprida, é fundamental uma mudança de visão das instituições (e/ou governo) sobre a importância de formações continuadas. A capacitação de profissionais bem treinados a conduzirem avaliações qualificadas de casos de violência sexual contra crianças e adolescentes não somente possibilita o cumprimento da Lei, mas atua como recurso protetivo aos seus profissionais e às vítimas atendidas.

A Etapa 2 deste estudo objetivou compreender quais os contextos de atuação (i.e., suporte à transferência), características do protocolo NICHD e aspectos do treinamento facilitaram/dificultaram a adesão à utilização do protocolo NICHD. As respostas às entrevistas contribuíram para a explicação da maneira pela qual os resultados de reação e consequentemente transferência de aprendizagem e impacto ocorreram e se relacionaram entre si. Os principais pontos apresentados pelos participantes como sugestões foram: a realização de treinamento continuado e, entrevista com crianças e adolescentes com algum tipo de deficiência. A Lei 13.431 (Brasil, 2017) sugere que as capacitações realizadas sobre a escuta ou depoimento de crianças e adolescentes vítimas ou testemunhas de violência ocorra preferencialmente de maneira continuada, contudo, o baixo suporte à transferência evidencia a dificuldade do cumprimento de tal sugestão.

Uma possibilidade de tornar o treinamento contínuo seria a supervisão ou revisão de pares. A literatura que aborda a utilização do protocolo NICHD indica que profissionais treinados devem ser supervisionados constantemente para a manutenção do conhecimento adquirido (La Rooy et al., 2015; Lamb et al., 2018; Lamb, 2016). Na tentativa de solucionar tal demanda foi criado um grupo de WhatsApp para cada turma de treinamento, contudo a adesão dos participantes não foi boa. Tal questão surgiu como um subtema na análise das entrevistas. Isso evidencia a necessidade do desenvolvimento de tecnologias de supervisão que sejam efetivas em colaborar com a manutenção da adesão dos participantes ao uso do Protocolo NICHD (Lamb, 2016). Outro fator que pode ter contribuído para a não adesão à supervisão e/ ou revisão de pares é o receio dos participantes em se expor ou falar sobre casos sigilosos. Portanto, as supervisões devem fazer parte do programa de treinamento e preferencialmente, deve-se desenvolver tais treinamentos nos moldes de tecnologias sociais de capacitação profissional tendo em vista o sigilo dos casos apresentados. Levanta-se a hipótese de que a supervisão e/ou revisão de pares serviria como fator protetivo à saúde ocupacional colaborando à adesão do Protocolo NICHD.

Outro tema que emergiu nas entrevistas realizadas sugere que o treinamento para o uso do protocolo aborde temas com crianças com algum tipo de deficiência. A Organização Mundial de Saúde (World Health Organization - WHO, 2011) relata que 15\% da população mundial tem algum tipo de deficiência, sendo que o risco de vitimização desta população é 50\% maior do que a população geral. Crianças com algum tipo de deficiência apresentam risco três vezes maior de serem vitimizadas e 4,56 vezes maior de serem negligenciadas (Jones et al., 2012). O desenvolvimento de estudos sobre a utilização do Protocolo NICHD no Brasil com população com algum tipo de deficiência deve fazer parte da agenda de estudos sobre o assunto (Aznar-Blefari \& Padilha, 2015).

Ao analisar as entrevistas, foi possível observar o subtema “Extensão do Protocolo NICHD” relacionado à aplicação deste. Alguns participantes argumentaram que a utilização do protocolo poderia causar desconforto ao entrevistador por ser longo. Caberia discutir aqui se tal desconforto advém da utilização do protocolo propriamente dito ou de aspectos estressantes do trabalho com vítimas de violência. Em outro tema observado, os participantes questionam a sobrecarga de trabalho devido às demandas do sistema judiciário. A literatura demonstra que o trabalho com vítimas de violência aumenta o risco do desenvolvimento de depressão, quadros de ansiedade, burnout, estresse, queda no desempenho do trabalho e o uso de estratégias de coping desadaptativas como cigarro e álcool (Elliot \& Daley, 2013; Sherwood et al., 2019)

As medidas clássicas para avaliação de impacto do treinamen- 
to (i.e., reação, aprendizagem e impacto do treinamento) possibilitam parcialmente a compreensão de aspectos necessários para a qualificação do treinamento para o uso do Protocolo NICHD. Nesse sentido, destaca-se a importância de métodos mistos que permitem analisar a efetividade de treinamentos de maneira global. Levanta-se a hipótese de que a melhora de pontos sugeridos nas entrevistas, como a supervisão continuada após o treinamento, maior aprofundamento em aspectos desenvolvimentais relacionados à capacidade de crianças e adolescentes prestarem depoimento sobre o que foram expostas, discussão de pontos específicos como entrevistas com crianças e adolescentes com algum tipo de deficiência possibilitaria uma a adesão maior ao uso do protocolo NICHD. Tais dados vão de encontro com o que a literatura internacional argumenta sobre o uso do protocolo (Lamb et al., 2008; Lamb et al., 2018; Lamb, 2016).

O objetivo do presente estudo foi avaliar o impacto de um treinamento presencial para o uso do protocolo NICHD. Resultados como os níveis de engajamento no trabalho, autoeficácia ocupacional em intervenções com populações vulneráveis, reação e impacto do treinamento (medidos em amplitude e transferência de aprendizagem) sugerem o impacto positivo do treinamento. Contudo, o estudo demonstrou que a adesão ao uso da técnica e a redução da resistência à mudança dos participantes depende de um treinamento continuado e supervisionado. A partir do momento que a Lei 13.431 (Brasil, 2017) determina que os profissionais que realizarão a escuta especializada ou o depoimento especial sejam capacitados continuamente e adotem protocolos de entrevista investigativa cientificamente validados faz-se fundamental o desenvolvimento de técnicas de treinamento efetivas. Para tanto, a avaliação de variáveis de saúde ocupacional como engajamento no trabalho e autoeficácia ocupacional antes do treinamento e efeitos do treinamento como reações, transferência de aprendizagem e impacto são necessárias. Desta forma, pode-se questionar a forma pela qual o Sistema Judiciário tem se adequado à Lei (quais as medidas utilizadas na avaliação da efetividade das entrevistas e consequentemente na implantação dos métodos de entrevista).

Mesmo com a importância dos achados apresentados, o estudo apresentou limitações como a falta de uma medida pré-teste avaliando a aprendizagem, a não mensuração da qualidade das entrevistas realizadas após a adoção do protocolo NICHD, o número elevado de turmas de capacitação em datas e contextos distintos. $\mathrm{Na}$ agenda de pesquisas sobre o uso do protocolo NICHD no Brasil deve constar: (1) Avaliação de eficácia do treinamento para o uso do protocolo NICHD comparando os achados com grupo controle; (2) Desenvolvimento de uma tecnologia que facilite a disseminação do conhecimento sobre o Protocolo NICHD em todo território brasileiro (e.g., ensino à distância).

\section{Referências}

Abbad, G. (1999). Um modelo integrado de avaliação do impacto do treinamento no trabalho - IMPACT (Tese de doutorado não-publicada). Curso de Pós-Graduação em Psicologia da Universidade de Brasília, Brasil.

Abbad, G., Pilati, R., Borges-Andrade, J. E., \& Sallorenzo, L. H. (2012). Impacto do treinamento no trabalho-medida em amplitude. Em G. S. Abbad, L. Mourão, P. P. M. Meneses, T. Zerbini, J. E. Borges-Andrade, \& R. VilasBoas (Eds.), Medidas de avaliação em treinamento, desenvolvimento e educação (pp. 145-162). Artmed.

Abbad, G. S., Zerbini, T., \& Borges-Ferreira, M. F. (2012). Medidas de reação a cursos presenciais. Em G. S. Abbad, L. Mourão, P. P. M. Meneses, T. Zerbini, J. E. Borges-Andrade, \& R. Vilas-Boas (Eds.), Medidas de avaliação em treinamento, desenvolvimento e educação (pp. 78 - 90). Artmed.

Abbad, G., Gama. A. L. G., \& Borges-Andrade, J. E. (2000). Treinamento: análise do relacionamento da avaliação nos níveis de reação aprendizagem e impacto no trabalho. Revista de Administracão Contemporânea, 4(3), 25-45. https://doi.org/10.1590/S1415-65552000000300003
Aznar-Blefari, C., \& Padilha, M. G. S. (2015). Capacitação para o uso do Protocolo NICHD em profissionais sul-brasileiros. Revista de Psicología, 24(1), 1-19. https://doi.org/doi: 10.5354/0719-0581.2015.37198

Bakker, A. B. (2011). An evidence-based model of work engagement. Current Directions in Psychological Science, 20(4), 265-269. https://doi. org/10.1177/0963721411414534

Bakker, A. B., \& Demerouti, E. (2007). The job demands-resources model: State of the art. Journal of Managerial Psychology, 22, 309-328. https://doi. org $/ 10.1108 / 02683940710733115$

Bakker, A. B., Demerouti, E., \& Sanz-Vergel, A. I. (2014). Burnout and work engagement: The JD-R approach. Annual Review of Organizational Psychology and Organizational Behavior, 1(1), 389-411. https://doi.org/10.1146/annurevorgpsych-031413-091235

Bell, B. S., Tannenbaum, S. I., Ford, J. K., Noe, R. A., \& Kraiger K. (2017). 100 Years of Training and Development Research: What We Know and Where We Should Go. Journal of Applied Psychology, 1-19. https://doi.org/10.1037/ apl0000142

Benson, M. S., \& Powell, M. B. (2015). Evaluation of a comprehensive interactive training system for investigative interviewers of children. Psychology, Public Policy, and Law, 21(3), 309-322. https://doi.org/10.1037/law0000052

Brasil (2017). Lei no 13.431 de 4 de abril de 2017. Recuperado de http://www. planalto.gov.br/ccivil 03/ ato2015-2018/2017/lei/L13431.htm

Braun, V., \& Clarke, V. (2006). Using thematic analysis in psychology. Qualitative Research in Psychology, 3(2), 77-101. https://doi. org/10.1191/1478088706qp063oa

Creswell, J. W., \& Clark, V. P. C. (2013). Pesquisa de métodos mistos. Porto Alegre: Penso.

Curado, C., Henriques, P. L., \& Ribeiro, S. (2015). Voluntary or mandatory enrollment in training and the motivation to transfer training. International Journal of Training and Development, 19(2), 98-109. https://doi.org/10.1111/ ijtd. 12050

Damásio, B. F., Habigznag, L. F., Freitas, C. P. P., \& Koller, S. H. (2014). Avaliação de processo de uma tecnologia social de capacitação profissional para psicólogos que atendem casos de violência sexual. Contextos Clínicos, 7(2), 207-219. https://doi.org/10.4013/ctc.2014.72.08

de Farias, B. G., Dutra-Thomé, L., Koller, S. H., \& Castro, T. G. (2020). Formulation of themes in qualitative research: logical procedures and analytical paths. Trends in Psychology, 1-12. https://doi.org/10.1007/s43076020-00052-0

Faller, K. C. (2015). Forty years of forensic interviewing of children suspected of sexual abuse, 1974-2014: historical benchmarks. Social Sciences, 4, 34-65. https://doi.org/10.3390/socsci4010034

Fletcher, L. (2015). Training perceptions, engagement, and performance: comparing work engagement and personal role engagement. Human Resource Development International, 19(1), 1-23. https://doi.org/10.1080/13678868.20 15.1067855

Freitas, C. P. P. (2013). Avaliação de impacto de uma tecnologia social para profissionais de psicologia que trabalham com vítimas de violência sexual. (Dissertação de mestrado não publicada). Universidade Federal do Rio Grande do Sul, Brasil.

Freitas, C. P. P., Silva, C. S. C., Damásio, B. F., Koller, S. H., \& Teixeira, M. A. P. (2016). Impact of Job-Related Well-Being on the Relationship of Self-Efficacy With Burnout. Paidéia, 26(63), 45-52. https://doi. org $/ 10.1590 / 1982-43272663201606$

Hackbarth, C., Lopes, N. R. L., \& Williams, L. C. A. (2018). Estudo piloto com profissionais sobre o uso do protocolo NICHD para investigar abuso sexual infantil (53-70) Em L. F. Habigzang, P. I. C. Gomide, \& G. M. Rocha (Orgs.), Psicologia forense: temas e práticas (pp. 53-70). Curitiba: Juruá.

Hackbarth, C., Williams, L. C. A., \& Lopes, N. R. L. (2015). Avaliação de capacitação para utilização do Protocolo NICHD em duas cidades brasileiras. Revista de Psicología, 24(1), 1-18. https://doi.org/10.5354/07190581.2015 .36916

Hamblin, A. C. (1978). Avaliação e controle do treinamento. McGraw-Hill do Brasil.

Jones, L., Bellis, M. A., Wood, S., Hughes, K., McCoy, E., Eckley, L., Bates, G., Mikton, C., Shakespeare, T., Officer, A., (2012). Prevalence and risk of violence against children with disabilities: a systematic review and metaanalysis of observational studies. Lancet, 380(9845), 899 - 907. https://doi. org/10.1016/S0140-6736(12)60692-8

Kirkpatrick, D. L. (1976). Evaluation of training. Em R. L. Craig (Org.), Training and development handbook (pp 18.1-18.27). McGraw-Hill.

La Rooy, D., Brubacher, S. P., Aromaki-Stratos, A., Cyr, M., Hershkowitz, I., Korkman, J., Myklebust, T., Naka, M., Peixoto, C. E., Roberts, K., P., Stewart, H., \& Lamb, M. E. (2015). The NICHD Protocol: a review of na internationally used evidence-based tool for training child forensic interviewers. Journal of Criminological Research, Policy and Practice, 1(2), 76-89. https://doi.org/10.1108/JCRPP_01-2015-0001

Lamb, M. E. (2016). Difficulties translating research on forensic interview practices to practitioners: finding water, leading horses, but can we get them to drink?. American Psychologist, 71, 710-718. https://doi.org/10.1037/ amp0000039 
Lamb, M. E., Brown, D. A., Hershkowitz, I., Orbach, Y., \& Esplin, P. W. (2018). Tell me what happened: questioning children about abuse. Chichester, UK: WileyBlackwell.

Lamb, M. E., Hershkowitz, I. Y., Orbach, W. E., \& Esplin, P. W. (2008). Tell me what happened: Structured investigative interviews of child victims and witnesses. WileyBlackwell.

Magnan, R. S., Vazquez, A. C. S., Pacico, J. C., \& Hutz, C. S. (2016). Normatização da versão Brasileiras da Escala Utrecht de Engajamento no Trabalho. Avaliacão Psicológica, 15(2), 133-140. https://doi.org/10.15689/ ap.2016.1502.01

Memon, M. A., Salleh, R., \& Baharom, M. N. R. (2016). The link between training satisfaction, work engagement and turnouver intention. European Journal of Training and Development, 40(6), 407-429. https://doi.org/10.1108/EJTD-102015-0077

Moreira, R., Abbad, G. S., Cualheta, L. P., \& Neiva, E. R. (2019). Força motivacional, suporte à transferência e impacto do treinamento no trabalho. Revista Psicologia: Organizacões e Trabalho, 19(2), 631-639. https://doi. org $/ 10.17652 / \mathrm{rpot} / 2019.2 .15939$

Oliveira, L. C., \& Rocha, J. C. (2017). Engajamento no trabalho: antecedentes individuais e situacionais e sua relação com a intenção de rotatividade. Revista Brasileira de Gestão de Negócios, 19(65), 415-431. https://doi. org/10.7819/rbgn.v19i64.3373

Pilati, R., \& Abbad, G. (2005). Análise fatorial confirmatória da Escala de Impacto do Treinamento no Trabalho. Psicologia: Teoria e Pesquisa, 21(1), 43 51. https://doi.org/10.1590/S0102-37722005000100007

Sampaio, N. S. P., Borges-Andrade, J. R., Bonatti, C. L. (2018). Aprendizagem no trabalho: pesquisa nas organizações públicas e privadas brasileiras. Psicología desde el Caribe (Número Especial), 1-21. https://doi.org/10.14482/ psdc.35.4.158.72

Schaufeli, W. B. (2014). What is engagement? Em C. Truss, R. Delbridge, K. Alfes, A. Shantz, \& E. Soane. (Eds.), Employee engagement in theory and practice (15-35). Routledge.

Schaufeli, W. B., Salanova, M., González-Romá, V., \& Bakker, A. B. (2002). The measurement of engagement and burnout: A two simple confirmatory factor analytic approach. Journal of Happiness Studies, 3(1), 71-92. https://doi. org/10.1023/A:1015630930326

Schoeb, G., Lafrenière-Carrier, B., Lauzier, M., \& Courcy, F. (2019). Measuring transfer of training: review and implications for future research. Canadian Journal of Administrative Sciences, 1-2. https://doi.org/10.1002/cjas.1577

Sherwood, L., Hegarty, S., Vallières, F., Hyland, P., Murphy, J., Fitzgerald, G., \& Reid, T. (2019). Identifying the key risk factors for adverse pychological outcomes among police officers a systematic literature review. Journal of Traumatic Stress, 00, 1-13. https://doi.org/10.10002/jts.22431

Souza, L. K. (2019). Pesquisa com análise qualitativa de dados: conhecendo a análise temática. Arquivos Brasileiros de Psicologia, 71(2), 51-67. Recuprado de http://pepsic.bvsalud.org/scielo.php?script=sci arttext\&pid=S1809-52672019000200005

Tamayo, N., \& Abbad, G. S., (2006). Autoconceito profissional e suporte à transferência e impacto do treinamento no trabalho. Revista de Administração Contemporânea, 10(3), 9 - 28. https://doi.org/10.1590/S1415$\underline{65552006000300002}$

Vazquez, A. C., Magnan, E. S., Pacico, J. C., Hutz, C. S., \& Schaufeli, W. B. (2015). Adaptação e validação da versão brasileira da Utrecht Work Engagement Scale. Psico-USF, 20(2), 207-217. https://doi.org/10.1590/1413$\underline{82712015200202}$

Williams, L. C. A., Padilha, M. G. S., Hackbarth, C., Blefari, C. A., \& Peixoto, C. E. (2014). Investigação de suspeita de abuso sexual infanto-juvenil: O protocolo NICHD. Temas em Psicologia, 22(2), 415-432. https://doi. org/10.9788/TP2014.2-12

World Health Organization (WHO, 2011). Global burden of disease. Disease and injury regional estimates. Recuperado de http://www.who.int/healthinfo/global burden disease/estimates regional/en/index.html3

\section{Informações sobre os autores:}

Os autores agradecem o apoio da Coordenação de Aperfeiçoamento de Pessoal de Nível Superior - Brasil (CAPES)

- Código de Financiamento 001

\section{Carlos Aznar-Blefari}

Pontifícia Universidade Católica do Rio Grande do Sul Av. Ipiranga, 6681, Prédio 11, $9^{\circ}$ andar, Sala 924 90619-000 Porto Alegre (RS), Brasil

E-mail: psicoaznar@gmail.com

\section{Marcela Schimaneski Lunardon}

E-mail: psicoschi@gmail.com

\section{Clarissa Pinto Pizarro de Freitas}

E-mail: freitas.cpp@gmail.com

\section{Luísa Fernanda Habigzang}

E-mail: habigzang.luisa@gmail.com 